\title{
Cloning and expression in Escherichia coli of a Streptomyces coelicolor A3(2) argCJB gene cluster
}

\author{
Zoe Hindle, ${ }^{1} \nmid$ Rowena Callis, ${ }^{1}$ Simon Dowden, ${ }^{2}$ Brian A. M. Rudd ${ }^{2}$ and \\ Simon Baumberg ${ }^{1}$
}

Author for correspondence: Simon Baumberg. Tel: +44532333080 . Fax : + 44532441175.

1 Department of Genetics, University of Leeds, Leeds LS2 9JT, UK

2 Glaxo Group Research Ltd, Greenford Road, Greenford, Middlesex UB6 OHE, UK
From a partial Sau3AI library of Streptomyces coelicolor A3(2) DNA in plJ916, two hybrid plasmids PGX1 and pGX2 were isolated that complemented S. coelicolor A3(2) or S. lividans arginine auxotrophs. Subcloning DNA from PGX1 in the Escherichia coli expression vector PRK9 containing the Serratia marcescens trp promoter gave rise to one plasmid, pZC2, that complemented $E$. coli argB, $C, E$ and $H$ auxotrophs, and another, pZC1, that complemented only the first three. The plasmids were markedly unstable in the various complemented hosts, to varying extents; pZC1 was characterized further as providing the stablest host/plasmid combinations. In vitro deletion of part of the vector's trp promoter did not affect complementation of the argB and $C$ auxotrophs, implying that the 5 . coelicolor A3(2) arg genes may be expressed from their own promoter. The trp promoter-less plasmids included isolates, such as pZC177, that had suffered extensive further deletion without loss of complementing ability. Extracts of an $E$. coli argE auxotroph carrying pZC177 showed ornithine acetyltransferase activity, indicating that the complementing gene is of the argJ type. The complementation properties of in vitro deletion derivatives of pZC177 indicated the gene order argC-J-B. Part of argC and the upstream region were sequenced; an ORF was identified whose predicted product showed appreciable homology with the E. coli and Bacillus subtilis ArgC polypeptide. Upstream of this ORF a consensus-type promoter and ribosome binding site could be discerned; overlapping its promoter was a sequence with homology to arginine operators in these two other organisms. An in vitro frameshift in argC had a polar effect on expression in $E$. coli of arg $J$ and $B$, suggesting that the three genes are transcribed in the same direction, possibly as an operon.

Keywords: Streptomyces coelicolor, argCJB gene cluster, cloning, expression, arginine auxotrophy

\section{INTRODUCTION}

Studies on the molecular genetics of streptomycetes have so far largely concentrated on their uniquely varied secondary metabolism. Primary metabolic systems, in contrast, whether catabolic, anabolic or anaplerotic, have received less attention, particularly from the viewpoint of

\footnotetext{
†Present address: Department of Biochemistry and Applied Molecular Biology, UMIST, Manchester M60 1QD, UK.

The EMBL accession number for the sequence reported in this paper is X66783.
}

regulation. This is perhaps surprising, since secondary metabolites are necessarily synthesized from primary metabolite precursors whose pool sizes and rates of formation and breakdown are likely to influence secondary metabolite yields. Catabolic systems that have been studied in detail include those for glycerol in Streptomyces coelicolor A3(2) (Smith \& Chater, 1988a, b) and galactose in S. lividans (Adams et al., 1988). Other amino acid pathways that have been examined in some detail are histidine biosynthesis in $S$. coelicolor A3(2) (Limauro et al., 1990) and proline synthesis, catabolism and transport and tryptophan biosynthesis (Hood $e t$ al., 1992). There is insufficient information, however, to tell 


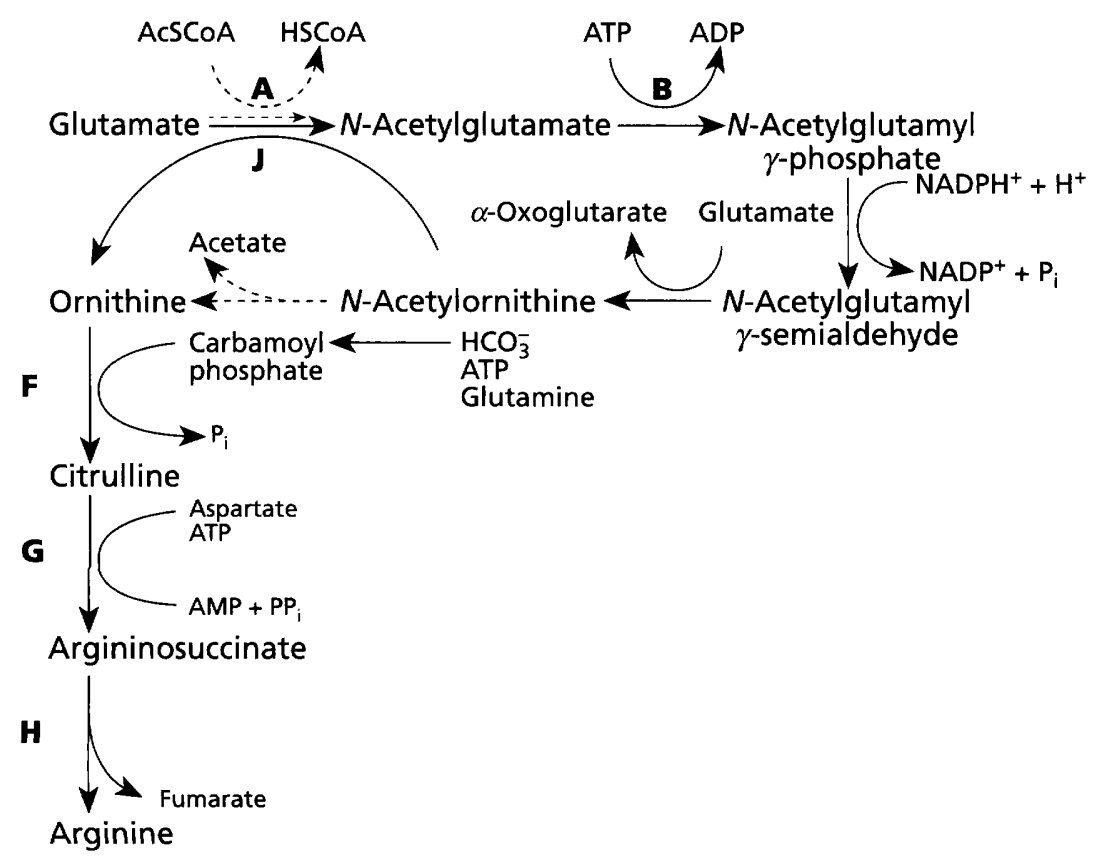

Fig. 1. Pathways of arginine biosynthesis in micro-organisms. Letters A-J refer to enzymes mediating each step, these being encoded by genes $\arg A$-arg $J$, respectively. For further information, see Cunin et al. (1986) and Glansdorff (1987). how the range of control devices (Baumberg, 1981) in streptomycetes compares to those of the enterobacteria or bacilli; or whether either in catabolic or anabolic systems there occur controls of gene expression analogous or even homologous to those found in other groups.

We have embarked on a study of arginine biosynthesis and catabolism in the model streptomycete $S$. coelicolor A3(2) to gain answers to such questions in this system, following on from work on the Bacillus subtilis system (Czaplewski et al., 1992, and references therein). The first step was to clone genes of arginine biosynthesis. This could in principle be done by complementation of streptomycete auxotrophs, since arginine auxotrophic markers $\arg A 1, \operatorname{argB} 2$ and $\arg C 4$ are available in $S$. coelicolor $\mathrm{A} 3(2)$ and arg- 8 in $S$. lividans. However, the only information available about these auxotrophs is their response to the arginine precursors ornithine and citrulline, leaving the biochemical lesions uncertain (see Fig. 1 for the arginine biosynthetic pathway); the $S$. coelicolor A3(2) locus designations $\arg A, B$ and $C$ (Hopwood et al., 1985) therefore do not correspond to steps in the pathway as denoted in Fig. 1. The $\arg A 1, C 4$ and $\arg -8$ mutants respond to ornithine or citrulline, whilst the $\operatorname{argB2}$ mutant responds only to arginine. As regards the map locations of arginine biosynthesis genes, the three $S$. coelicolor A3(2) mutant alleles are shown as mapping close together on the linkage map (Hopwood et al., 1985). A further piece of information relates to the $\arg G$ gene encoding argininosuccinate synthetase, which may be deleted in a variety of streptomycetes as part of vast deletions (sometimes of Mbp), sometimes also associated with large amplifications (Schrempf, 1991; Altenbuchner \& Eichenseer, 1991; Cullum et al., 1991; Piendl et al., 1991). As no other arginine biosynthesis gene has been unequivocally shown to be deleted in such cases, it seems likely that $\arg G$ is located separately from other genes of arginine bio- synthesis; an implication is that the gene affected in argB2 is $\operatorname{argH}$, encoding argininosuccinate lyase. The $S$. coelicolor A3(2) arg $G$ gene has been cloned and sequenced (Ishihara et al., 1985, 1991), as has that from S. lavendulae (Ogawara et al., 1993).

As indicated in Fig. 1, there are two different forms of the pathway between glutamate and ornithine (Cunin et al., 1986). In Escherichia coli and other enterobacteria, there are separate steps for synthesis of $N$-acetylglutamate from glutamate and acetyl-CoA, catalysed by $N$-acetylglutamate synthase (encoded by $\arg A$ ), and cleavage of $N$ acetylornithine to ornithine and acetate, catalysed by acetylornithinase (encoded by argE). In many groups, including pseudomonads and, as recently shown, bacilli (Sakanyan et al., 1992, 1993; O'Reilly et al., 1994), there is an ornithine acetyltransferase (whose gene is conventionally termed $\mathrm{arg} /$ ) which catalyses the transfer of an acetyl group from $N$-acetylornithine to glutamate, the ArgA enzyme having only an anaplerotic role. An early report (Udaka, 1966), so far unconfirmed, suggested that $S$. griseus also possesses an Arg J activity. The situation in $S$. coelicolor A3(2), whilst likely to resemble that in S. griseus, has not been investigated.

We describe here the cloning of S. coelicolor A3(2) genes of arginine biosynthesis and their introduction into defined E. coli auxotrophs to identify them precisely and map them with respect to each other, an approach used successfully in cloning arginine biosynthesis genes of Bacillus subtilis (Mountain et al., 1986). We also report part of the sequence of one gene, $\arg C$, and its upstream region. In parallel with this work, Ludovice et al. (1992) have reported the cloning, sequencing and determination of the transcription startpoint of $\arg C$ from $S$. clavuligerus, in which organism the ornithine/arginine pathway provides precursors for clavulanic acid biosynthesis. These authors also showed linkage to $\arg C$ of an $S$. clavuligerus 
gene complementing an E. coli argE mutation, and that the arginine auxotrophy of $S$. lividans 1674 is due to an $\arg C$ lesion. A preliminary account containing elements of both this work and that of Ludovice et al. (1992) appeared in Padilla et al. (1991).

\section{METHODS}

Bacterial strains and plasmids. S. coelicolor A3(2) prototroph M145 and arginine auxotrophs M124 ( $\arg A 1)$, J12281 (argB2) and JI2345 (argC44), and S. lividans JI1674 (arg-8), as well as the SCP2*-based plasmid vector pIJ916, were provided by D. A. Hopwood and colleagues, John Innes Institute. The E. coli strains all derive from this laboratory; $\mathrm{Hsd}^{-}$arginine auxotrophs $\mathrm{XA} 4(\arg A), \mathrm{XB} 25(\operatorname{argB}), \mathrm{XC33}(\arg C), \mathrm{XS1D} 2(\arg E), \mathrm{XF}$ $(\operatorname{argF}), \mathrm{XG} 31(\operatorname{argG}), \mathrm{XH11}(\operatorname{argH}), \mathrm{X} 190(\operatorname{car} A)$ and X Jef8 (carB) are described in Mountain et al. (1984), and 6P in Bretscher \& Baumberg (1976). The E. coli plasmid vectors, also this laboratory's stocks, were pRK9 (Lim et al., 1989) and pUC18 (Messing \& Vieira, 1982). The prototrophic Neurospora crassa strain 74R23-1A was kindly provided by A. Radford (Department of Genetics, University of Leeds).

Media and growth conditions. Media for E. coli were as in Maniatis et al. (1982) except for minimal media which were as in Mountain et al. (1984). 2TYAmp and Min Amp refer to nutrient (2TY) or minimal media used for plasmid marker selection with ampicillin at $50 \mu \mathrm{g} \mathrm{ml}^{-1}$; MinAmp was always supplemented with any auxotrophic requirements other than arginine, in particular the disodium succinate (at $0.5 \%$ ) needed by strain XS1D2 which carries a $p p c-a r g E$ deletion. E. coli strains were grown at $37^{\circ} \mathrm{C}$ except where an arginine auxotroph was being complemented, when growth was at $30^{\circ} \mathrm{C}$. Media and growth conditions for Streptomyces strains were as in Hopwood et al. (1985).

Enzyme assays. Ornithine acetyltransferase was assayed by the method of Denes (1970). $N$-acetylornithinase was assayed by the method of Vogel \& Bonner (1956). Protein was estimated as described by Bradford (1976).

Isolation, restriction and ligation of DNA. Plasmid DNA was isolated from E. coli by the method of Birnboim \& Doly (1979). Chromosomal DNA was isolated from $S$. coelicolor A3(2) as in Hopwood et al. (1985); plasmid DNA was isolated from Streptomyces strains either according to Hopwood et al. (1985) or by the method of Birnboim \& Doly (1979). Restriction endonucleases, DNA ligase, mung bean single-strand nuclease and Klenow fragment of DNA polymerase were obtained from standard suppliers and used according to makers' recommendations.

Techniques. Gel electrophoresis, elution of DNA fragments from gels, preparation of radioisotope-labelled probes by nicktranslation, and Southern blot hybridization were carried out essentially as in Maniatis et al. (1982). Bacterial transformation was carried out according to Maniatis et al. (1982) (E. coli) or Hopwood et al. (1985) (Streptomyces strains).

DNA sequencing. The $S a l(0)-S m a I(1)$ fragment of pZH1801 and the PvuII(1)-SmaI(1) fragment of pZC1773 (see Fig. 3a) were each cloned into sequencing vectors $\mathrm{M} 13 \mathrm{mp} 8$ and M13mp19 (Norrander et al., 1983). Sequencing was carried out with the Pharmacia T7 Polymerase Sequencing Kit according to the maker's instructions. To permit sequencing across the

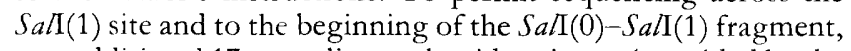
two additional 17-mer oligonucleotide primers (provided by the Biotechnology Unit, University of Leeds) were employed : these corresponded to bases 272-288 (strand shown) and 324-340 (complementary to strand shown) of Fig. 5.
Computer-aided sequence analysis. This was carried out by use of the Leeds University OWL database and associated software (Akrigg et al., 1988).

\section{RESULTS}

\section{Cloning of $S$. coelicolor A3(2) arginine biosynthesis genes in streptomycetes and their subcloning in $E$. coli by complementation of arginine auxotrophs}

A gene library of the prototrophic $S$. coelicolor A3(2) strain M145 was produced by partially digesting total DNA with Sau3AI and ligating fragments of size $8 \mathrm{kbp}$ or above into the $B g l \mathrm{II}$ site of the SCP2*-based vector pIJ916 (Hopwood et al., 1985). The resulting hybrids were transformed into the $S$. coelicolor A3(2) arginine auxotrophs M124, JI2281 and JI2345, and S. lividans JI1674, initially with selection for thiostrepton resistance; transformants were then replica plated on minimal medium without arginine to detect complementation. In this way, two plasmids, pGX1 and pGX2, were obtained that complemented streptomycete arginine auxotrophs: pGX1 complemented all four, pGX2 all except JI2345, from which it may be concluded that pGX1 carries argH (see Introduction), whilst pGX2 does not. Single and double digests with a number of restriction endonucleases (data not shown) yielded the restriction maps of the two plasmids shown in Fig. 2. They have inserts of $17.2 \mathrm{kbp}$ and $12.9 \mathrm{kbp}$, respectively, with an overlap of about $10 \mathrm{kbp}$. The orientation of insert DNA in pGX1 with respect to the vector is opposite to that in pGX2.

We proceeded by subcloning DNA from pGX1 in an E. coli vector. It is known that streptomycete genes frequently express at a low level or not at all from their own promoters in E. coli (see discussion in Lim et al., 1989). We therefore used the expression vector $\mathrm{pRK} 9$ which has been used successfully before for a similar purpose, the subcloning of the S. griseus aphD gene (Lim et al., 1989); it is essentially pBR322 with the EcoRI-BamHI fragment replaced by a fragment containing the Serratia marcescens trp promoter. pGX1 was partially digested with Sau3AI and the fragments cloned into the BamHI expression site of pRK9. The ligation mixture was transformed into the four $E$. coli arg auxotrophs XA4, XB25, XC33 and $\mathrm{XS1D} 2$, carrying lesions in $\arg A, B, C$ and $E$, respectively (Mountain et al., 1984). Transformants were selected on 2TYAmp and replicated on to MinAmp plates. Three $\mathrm{Arg}^{+}$isolates were obtained with $\mathrm{XC33}$ as recipient and none with the other three strains; the plasmids in these three isolates were termed $\mathrm{pZC1}, \mathrm{p} Z \mathrm{C} 2$ and $\mathrm{pZC} 3$. Preliminary restriction analysis of these plasmids (results not shown) indicated that they contained inserts of about 30,31 and $17 \mathrm{kbp}$, respectively. Colonies of XC33 carrying pZC1 and pZC2 grew to reasonable size in $2-3 \mathrm{~d}$, whilst those with pZC3 took about $5 \mathrm{~d}$. For this reason, only pZC1 and pZC2 were studied further.

\section{Characteristics of pZC1 and pZC2: instability and complementation of further $E$. coli auxotrophs}

When XC33(pZC1) or XC33(pZC2) were cultured in 2TYAmp, additional smaller plasmids were found, at 


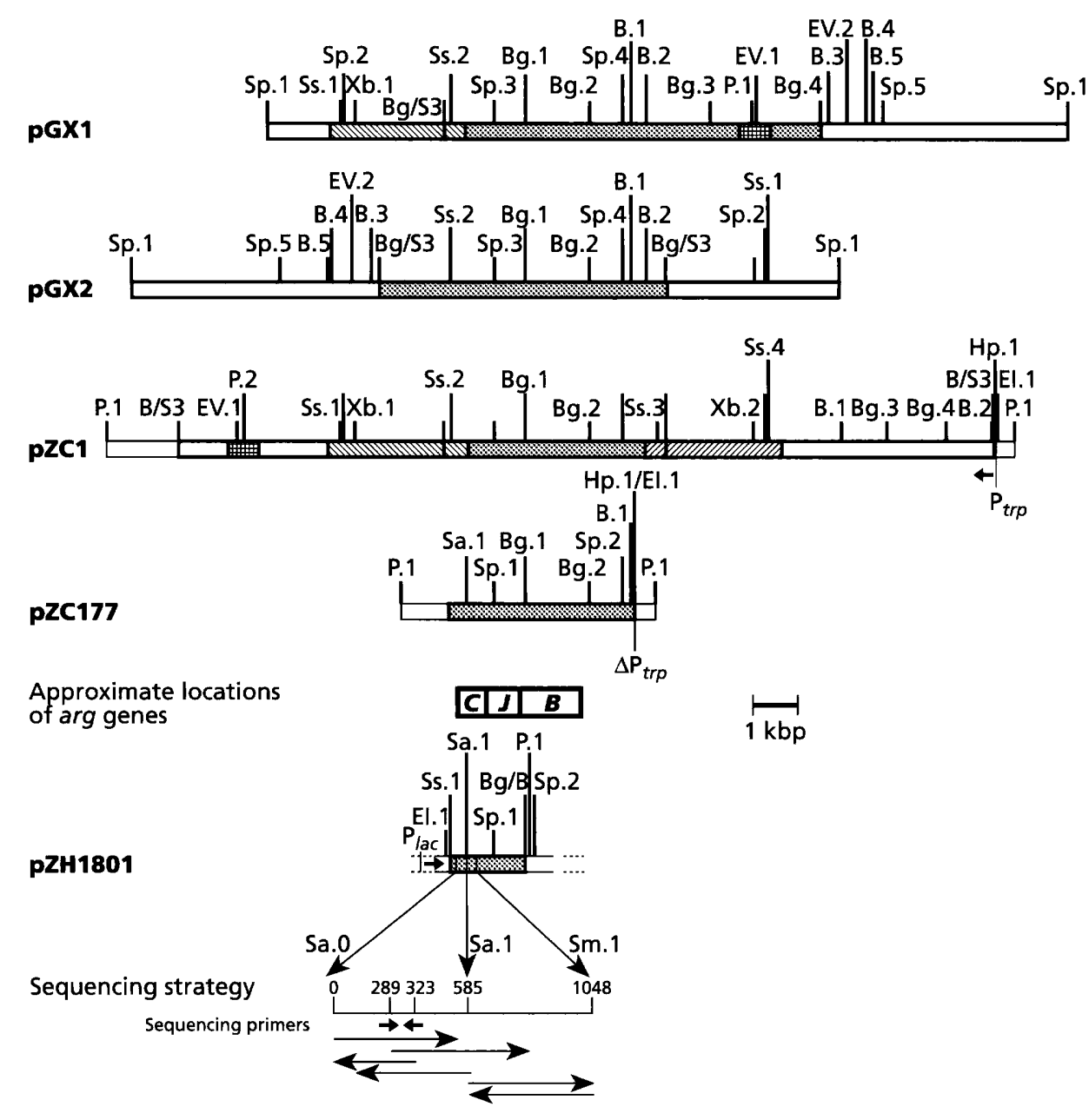

Fig. 2. Restriction maps and other details of plasmids pGX1, pGX2, pZC1, pZC177 and pZH1801. Stippled boxes, $S$. coelicolor chromosomal DNA; bold-outline open boxes, SCP2*-based vector DNA; thin-outline open boxes, pBR322-based vector DNA. Hatched boxes refer to a sequence present in one copy in pGX1 and apparently in two copies, in inverse orientation (shown by hatching in opposite direction), in pZC1. Cross-squared boxes refer to a sequence apparently displaced in pZC1. For pZH1801, only the insert and immediately surrounding regions (comprising the lac promoter and polylinker) are shown. The scale of the restriction map is as shown. Abbreviations of restriction enzymes: $\mathrm{B}, \mathrm{BamHI} ; \mathrm{Bg}$, Bglll; El, EcoRl; EV, EcoRV; Hp, Hpal; P, Pstl; S3, Sau3Al; Sa, Sall; Sp, Sphl; Ss, Sstl; Xb, Xbal. The numbering of sites is described in the text. Sites in pZC1 which are presumed to exist by similarity to the other plasmids but which have not actually been checked are indicated with lines but no lettering. The positions of the trp and lac promoters in pZC1 and pZH1801, respectively, are shown, arrowheads pointing in the direction of transcription. Approximate locations of the $\arg C J B$ genes are based on data shown in Fig. 3 and described in the text. The strategy for sequencing part of the insert in $\mathrm{pZH} 1801$ is shown together with the positions of internal sequencing primers used.

least some of which were unable on isolation to transform $\mathrm{XC} 33$ to $\mathrm{Arg}^{+}$(results not shown). The smaller plasmids had presumably arisen by deletion and/or rearrangement as a result of which the presumed cloned $S$. coelicolor A3(2) $\arg C$ gene either had been deleted completely or in part, or could no longer be expressed. On culture of XC33(pZC2) in MinAmp, pZC1 appeared to be stable. On culture of XC33(pZC2) in MinAmp, however, two plasmids could be discerned, one corresponding in size and restriction pattern to the original pZC2 and the other to a deletion derivative of size about $25 \mathrm{kbp}$; the latter, termed pZC201, could retransform XC33 to $\mathrm{Arg}^{+}$. On culture of $\mathrm{XC33}(\mathrm{pZC} 201)$ in MinAmp, the plasmid appeared to be stable.
Plasmids pZC1, pZC2 and pZC201 were used to transform others of the complete set of $\mathrm{Hsd}^{-} E$. coli arg auxotrophs (Mountain et al., 1984), namely XA4 $\left(\arg A^{-}\right)$, $\mathrm{XB} 25\left(\arg B^{-}\right), \mathrm{XS1D} 2\left(p p c^{-} \operatorname{argE^{-}}\right), \mathrm{XF}\left(\operatorname{argFI} I^{-}\right), \mathrm{XG} 31$ $\left(\arg G^{-}\right), \mathrm{XH11}\left(p p c^{-} \operatorname{argEC} C \mathrm{HH}^{-}\right), \mathrm{X} 190\left(\mathrm{car} A^{-}\right)$and X Jef 8 $\left(\right.$ car $\left.B^{-}\right)$. Transformants were again initially selected on 2TYAmp and replicated to MinAmp to check for Arg ${ }^{+}$. These tests showed that pZC1 could complement argB and $E$ mutations, and pZC201 the $\arg B$ mutation, in addition to $\arg C$, whilst pZC2 could complement $\arg B, E$ and $H$ mutations. In all cases where complementation was observed, less than $100 \%$ of transformants selected on 2TYAmp gave $\mathrm{Arg}^{+}$colonies when replicated on MinAmp. Plasmid DNA was isolated from the various 
$\mathrm{Amp}^{\mathbf{R}} \mathrm{Arg}^{+}$transformants, cut with PvuII or Bg/II, and electrophoresed. XB25(pZC1) yielded the equivalent of (in the sense of giving the same restriction pattern as) $\mathrm{pZC1}$, and XB25(pZC2) the equivalent of $\mathrm{pZC} 201$; XS1D2(pZC1) yielded only deletion derivatives, $X S 1 D 2(p Z C 2)$ the equivalent of $\mathrm{pZC201}$; and XH11(pZC2) yielded the equivalents of either $\mathrm{pZC} 2$ alone, pZC201 alone, or both. Whereas complementation of $\mathrm{XB25}$, like that of XC33, gave colonies that reached reasonable size in 2-3d, XS1D2(pZC1) or (pZC2) growing on MinAmp took 9-11 d to reach a similar size.

These results imply that pGX1 carries the $S$. coelicolor A3(2) analogues of $\arg B, C, H$ and either $E$ or $J$, the latter being presumably capable of complementing an E. coli $\operatorname{argE}$ mutation (see below). At the cost of not proceeding further with the $\operatorname{argH}$ gene, it was decided at this point to concentrate on $\mathrm{pZC1}$ as being the most stable of the plasmids.

\section{Restriction analysis of plasmid pZC1}

Single and double digests of pZC1 with a variety of restriction enzymes (results not shown: see Hindle, 1990) provided the restriction map of this plasmid shown in Fig. 2. Comparison with the map of $\mathrm{pGX} 1$ suggests that a number of non-contiguous fragments of pGX1 had combined to give $\mathrm{pZC1}$, as noted in the figure legend, and also that one region of pGX1 was present in duplicate in $\mathrm{pZC1}$, in tandem but with inverse orientation: the $S_{s t} \mathrm{I}(1)-S_{s t \mathrm{I}}(2)$ and $S_{s t} \mathrm{I}(3)-S_{s t} \mathrm{I}(4)$ fragments of $\mathrm{pZC1}$ probably correspond to $S_{s t} \mathrm{I}(1)-S_{s t} \mathrm{I}(2)$ of $\mathrm{pGX} 1$.

\section{From what promoter are the arginine biosynthesis genes transcribed in pZC1?}

It is of interest to know whether in pZC1 the arg genes are transcribed from their own promoter or from the trp promoter (or any other) in the vector. We have in previous cases (Hercomb et al., 1987; Lim et al., 1989) shown by deletion of part of a vector promoter that expression of cloned Streptomyces genes was from that promoter and not from their own. pZC1, as with the hybrid plasmids described in Lim et al. (1989) which are also pRK9 derivatives, has only single EcoRI and $H p a \mathrm{I}$ sites, the latter being within the trp promoter and the former just upstream of this. The sequence resulting from deletion of the DNA between these sites is predicted to be incapable of functioning as a consensus promoter (Lim et al., 1989). pZC1 was therefore cleaved with EcoRI, 5' overhangs removed with mung bean nuclease, cleaved with $H p a I$ and re-ligated the ligation mixture being transformed into XC33. Thirty-seven $\mathrm{Amp}^{\mathrm{R}}$ transformants were obtained on 2TYAmp. Of these, eight proved to be $\mathrm{Arg}^{+}$when tested on MinAmp but contained plasmids that had lost both sites; the $\mathrm{Arg}^{-}$transformants tested contained deleted versions of pZC1. On restriction analysis (not shown), three of the eight $\mathrm{Arg}^{+}$transformants were indistinguishable from $\mathrm{pZC1}$ except for the absence of the two restriction sites. Of the remainder, three appeared identical to each other but were derivatives of pZC1 that had suffered large deletions, being only about $12 \mathrm{kbp}$ in size. These three, however, like pZC1 complemented the $\arg B$ mutation of $\mathrm{XB} 25$ as well as the $\arg C$ of XC33. With these smaller plasmids, it was found that $100 \%$ of transformants of either strain initially selected as $\mathrm{Amp}^{\mathbf{R}}$ on 2TYAmp proved also to be $\mathrm{Arg}^{+}$on MinAmp, an improvement on complementation with pZC1 or pZC2 as noted above.

The simplest interpretation of the properties of the trp promoter deletions is that the cloned $S$. coelicolor A3(2) arg genes are not expressed from this promoter, and are either transcribed from some other vector promoter or from their own. Since pZC1 is about $41 \mathrm{kbp}$, making it unwieldy to work with, all further work was carried out on one of the $12 \mathrm{kbp}$ plasmids, termed pZC177. pZC177 also transforms the $\operatorname{argE}$ auxotroph XS1D2 to $\mathrm{Arg}^{+}$; XS1D2(pZC177) colonies grew on MinAmp within $2 \mathrm{~d}$, considerably faster than XS1D2(pZC1).

\section{Location of arginine biosynthesis genes within pZC177}

Single (sometimes partial) and double digests of pZC177 with a variety of restriction enzymes (results not shown: see Hindle, 1990) provided the restriction map of this plasmid shown in Figs 2 and 3(a). It may be noted that since the PvuII(1)-SalI(1) fragment of pZC177 is about the size expected for the small $P v u I I-S a l$ fragment of pRK9, the SalI(1) site of pZC177 might lie in vector DNA; in fact, it became apparent from DNA sequencing (see below) that this site lies within the $S$. coelicolor A3(2) insert DNA, the SalI site in the vector pRK9 evidently having been deleted. Comparison of the restriction maps of pZC177 and pGX1 (Fig. 2) suggested that the $S p h \mathrm{I}(1)-S p h \mathrm{I}(2)$ fragment of $\mathrm{pZC177}$ corresponded to the $S p b \mathrm{I}(3)-S p h \mathrm{I}(4)$ fragment of $\mathrm{pGX} 1$; it seemed probable therefore that the $S a l \mathrm{I}(1)-S_{p} b \mathrm{I}(2)$ region of $\mathrm{p} Z \mathrm{C} 177$ was colinear with the $S_{s t} \mathrm{I}(2)-S p h \mathrm{I}(4)$ region of $\mathrm{pGX} 1$. This was checked by Southern blot hybridizations using the $1.28 \mathrm{kbp} S a l \mathrm{I}(1)-S p b \mathrm{I}(1)$, the $1.5 \mathrm{kbp} S p b \mathrm{I}(1)-B g / \mathrm{II}(1)$, the $2.67 \mathrm{kbp} B g l \mathrm{II}(1)-B g l \mathrm{II}(2)$, and the $1.6 \mathrm{kbp} B g / \mathrm{II}(2)-$ $S p h I(2)$ fragments of pZC177 as probes against cleaved pGX1 and $S$. coelicolor M145 chromosomal DNA. The bands visible in the resulting autoradiographs (results not shown; see Hindle, 1990) were consistent with colinearity between the $S a l I(1)-S p h I(2)$ region of $\mathrm{pZC177}$, the $S_{s t} \mathrm{I}(2)-P s t \mathrm{I}(1)$ region of $\mathrm{pGX} 1$, and a region of genomic DNA of $S$. coelicolor M145 equivalent to the latter.

To determine where within pZC177 lie the genes complementing the arginine auxotrophic mutations in XB25, $\mathrm{XC} 33$ and XS1D2, a number of derivatives were constructed by in vitro deletion. These were (Fig. 3a): pZC1771, in which the $B g / \mathrm{II}(1)-B g / \mathrm{II}(2)$ fragment was deleted; pZC1772, in which the $B g l \mathrm{II}(2)-B a m \mathrm{HI}(1)$ fragment was deleted; and pZC1773, in which the SphI(1)$S p h \mathrm{I}(2)$ fragment was deleted. An additional construct was pZC1774, in which the $S p b \mathrm{I}(1)-B g / \mathrm{II}(1)$ fragment was

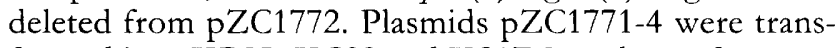
formed into XB25, XC33 and XS1D2, and transformants tested for growth on minimal medium lacking arginine; Fig. $3 \mathrm{a}$ indicates whether or not complementation was 


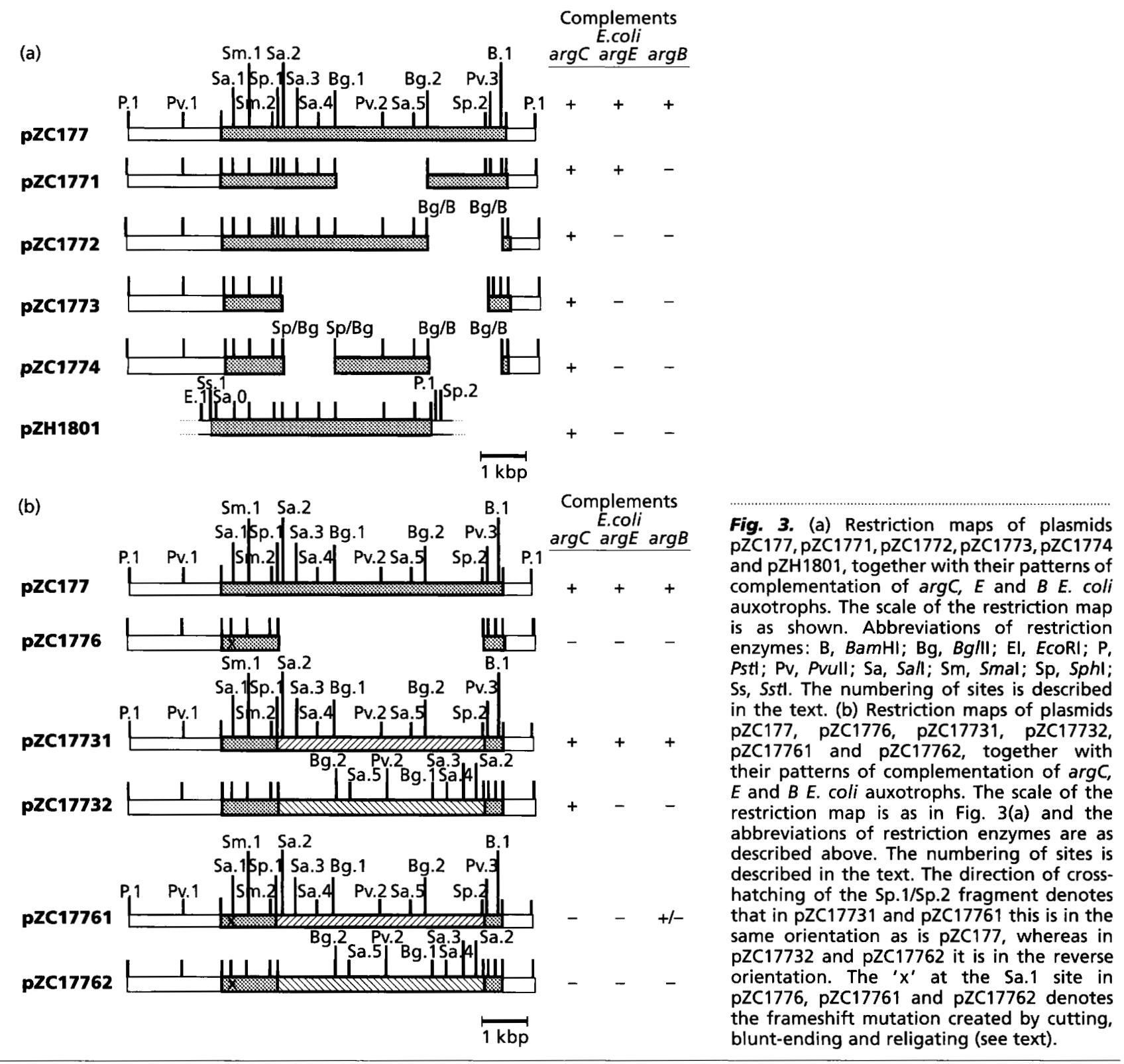

observed. The results clearly indicate a gene order C-' $E$ '$B$, where ' $E$ ' indicates that at this stage it cannot be said whether the complementing gene is an $\operatorname{argE}$ or an $\arg J$.

Further constructs made in part for sequencing (see below) were pZH1801 and pZH1901. In these, the $3.54 \mathrm{kbp} S s t \mathrm{I}(2)-B g / \mathrm{II}(1)$ fragment of pGX1 was ligated

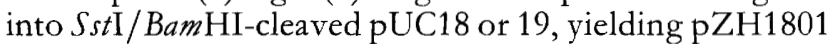
(Figs 2 and 3a) and pZH1901. Restriction analysis of pZH1801 revealed a further $S_{a l}$ I site between the $S_{s t} \mathrm{I}(2)$ site and the $S a l$ site corresponding to the $S a l I(1)$ site of pZC177. This new SalI site, which is absent from pZC177 and its derivatives, was termed $\operatorname{SalI}(0)$ (Figs 2 and $3 a$ ). Both pZH1801 and 1901 complemented XC33 and XS1D2; since this occurred with either orientation of the fragment with respect to the vector's lac promoter, expression in at least one orientation must rely on the insert's arg promoter, or an uncharacterized vector promoter.

\section{argE or args?}

As noted in the Introduction, it has been reported by Udaka (1966) that $S$. griseus possesses an ornithine acetyltransferase activity and therefore probably uses the cyclic pathway of ornithine synthesis; it might be expected that $S$. coelicolor A3(2) will be similar. To test this, extracts of XS1D2 carrving pZC177, with, as controls, XS1D2 carrying the vector $\mathrm{pRK}$, the $\arg \mathrm{E}^{+}$E. coli $\mathrm{K}-12$ strain $6 \mathrm{P}$ (Bretscher \& Baumberg, 1976) which possesses only $\operatorname{argE}$, and an $\mathrm{arg}^{+}$Neurospora crassa, which employs the cyclic pathway and therefore possesses argl (Davis, 1986), were assayed for their ability to convert acetylornithine to ornithine either in the presence of glutamate (which 
Table 1. Specific activities of ornithine acetyltransferase and acetylornithinase in $E$. coli strains with and without cloned $S$. coelicolor genes and in $N$. crassa

Cultures were grown in MinAmp (E. coli) or Vogel's minimal medium (N. crassa). XS1D2 cultures were supplemented with succinate and in the case of XS1D2 with arginine $\left(100 \mu \mathrm{g} \mathrm{ml}^{-1}\right)$ also. Specific activities are averages of two estimations made for different cultures.

\begin{tabular}{|lcc|}
\hline Strain & \multicolumn{1}{c|}{$\begin{array}{c}\text { Enzyme activity } \\
\text { [units (mg protein) }\end{array}$} \\
\cline { 2 - 3 } & $\begin{array}{c}\text { Ornithine } \\
\text { acetyltransferase }\end{array}$ & Acetylornithinase \\
\hline XS1D2(pRK9) & $<5$ & $<5$ \\
XS1D2(pZC177) & 15 & $<5$ \\
6P & $<5$ & 970 \\
N. crassa 74R23-1A & 1600 & 460 \\
\hline
\end{tabular}

measures both $\operatorname{ArgE}$ and $\operatorname{ArgJ}$ ) or in its absence (which measures only ArgE). The results are given in Table 1, from which it is seen that pZC177 confers on XS1D2 an ornithine acetyltransferase activity, confirming the conclusion of Udaka (1966). The low level of glutamateindependent activity found together with the ArgJ activity in the $N$. crassa extract may be non-specific, and seems to be general in microbial eukaryotes (see De Deken, 1963, for Saccharomyces cerevisiae). We can therefore say that the gene order in pZC177 is argC-J-B.

\section{Sequence analysis of $\arg C$ and its upstream region}

The PvuII(1)-SalI(2) fragment from pZC1773 (as illustrated for pZC177 in Fig. 3a) and the SalI(0)-SmaI(1) fragment from pZH1801 (Figs 2 and $3 a$ ) sequenced as in Methods, according to the strategy depicted in Fig. 2. The pZH1801 sequence, all of which was obtained on both strands, is shown in Fig. 4; the pZC1773 sequence showed 25 bp corresponding to pBR322 nucleotides 1027-1003 (Balbas et al., 1986), followed by nucleotide 246 of the sequence shown in Fig. 4. An ORF within the former was identified whose predicted translation product showed homology with the $E$. coli, B. subtilis and $S$. clavuligerus ArgC polypeptides (Parsot et al., 1988; Smith et al., 1990; Ludovice et al., 1992). An alignment of these four and homologous sequences from Saccharomyces cerevisiae and an Anabaena sp. are to be found in Ludovice et al. (1992), together with a detailed comparison of the sequences. Comparison of Figs 2 and 4 makes clear that the direction of transcription of $\arg C$ is opposite to that directed by the trp promoter of the $\mathrm{pRK} 9$ vector, consistent with this gene being expressed from its own (or possibly an uncharacterized vector) promoter.

\section{Direction of transcription of arg $J$ and $\arg B$}

To gain information on this point, a frameshift mutation was introduced into $\arg C$ and the effects on ability of the downstream $\arg J$ and $B$ to complement XS1D2 and XB25

\begin{abstract}
Sa. 0 $\begin{array}{ccccccc}10 & 20 & 30 & 40 & 50 & 60 & 70\end{array}$ $\begin{array}{rrrrrr}80 & 90 & 100 & 110 & 120 & 130\end{array}$

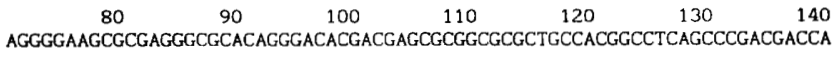

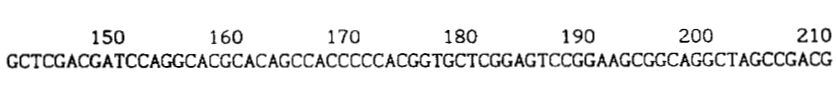

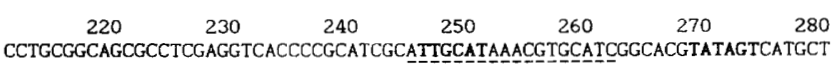

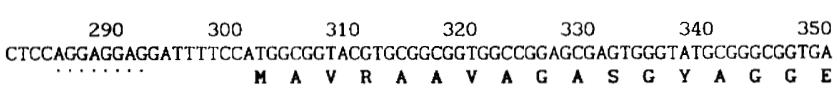

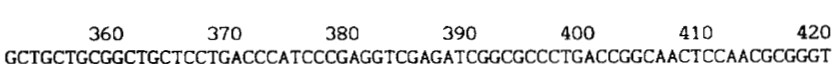
$\begin{array}{lllllllllllllllllllllll}\mathbf{L} & \mathbf{L} & \mathbf{R} & \mathbf{L} & \mathbf{L} & \mathbf{L} & \mathbf{T} & \mathbf{H} & \mathbf{P} & \mathbf{E} & \mathbf{V} & \mathbf{E} & \mathbf{I} & \mathbf{G} & \mathbf{A} & \mathbf{L} & \mathbf{T} & \mathbf{G} & \mathbf{N} & \mathbf{S} & \mathbf{N} & \mathbf{A} & \mathbf{G}\end{array}$ $\begin{array}{lllllll}430 & 440 & 450 & 460 & 470 & 480 & 490\end{array}$

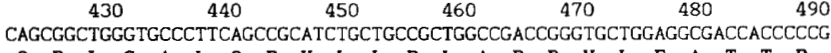

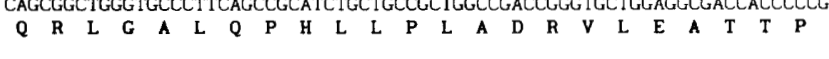

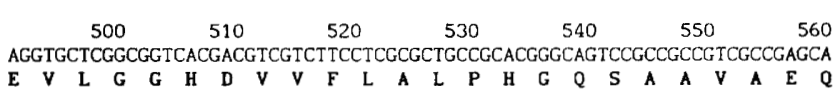

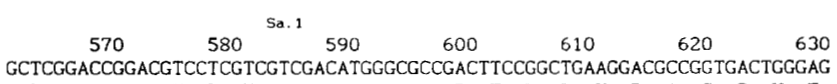

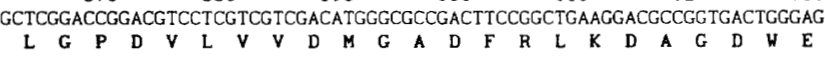

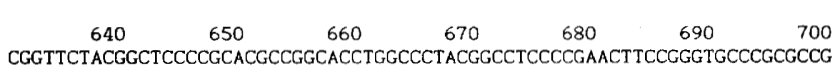

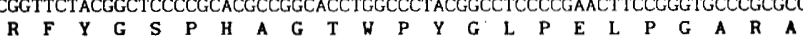
$\begin{array}{lllllll}710 & 720 & 730 & 740 & 750 & 760 & 770\end{array}$

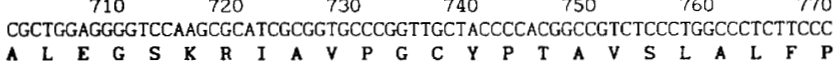

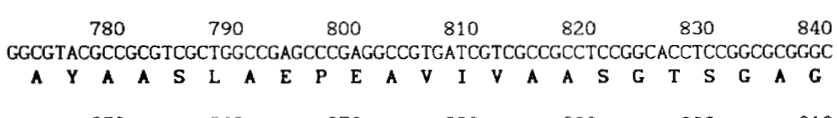
$\begin{array}{ccccccc}850 & 860 & 870 & 880 & 890 & 900 & 910 \\ \text { AAGGCGGCCAAGCCGCACCTGCTGGGCAGCGAGGTCATGGGCTCCATGTCGCCGTACGCCGTCGGCGGCG }\end{array}$

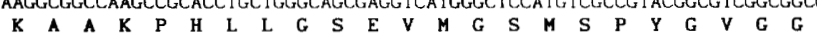

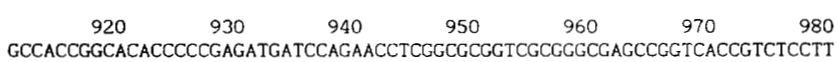

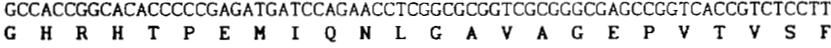

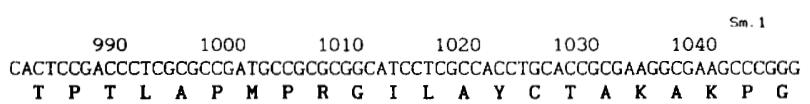

Fig. 4. DNA sequence of the Sall(0)-Smal(1) fragment of pZH1801, with the predicted amino acid sequence encoded by an ORF. The positions of restriction sites $\mathrm{Sall(0),Sa/I(1)}$ and Smal(1) referred to in Fig. 2 are indicated. Bases in bold type, 246-251 and 269-274, and dot-underlined bases 285-292 are putative $-35 /-10$ promoter boxes and the ribosome binding site, respectively. Underlined bases, 245-262, indicate the 18 bp sequence with homology to known arginine operator sites of $E$. coli and $B$. subtilis.
\end{abstract}

determined. pZC1773 (Fig. 3a) was cleaved with Sall, the 5 'overhangs filled in with Klenow enzyme, and the blunt ends religated to generate $\mathrm{pZC1776.} \mathrm{This} \mathrm{treatment,} \mathrm{as}$ predicted, generated a new $P v u \mathrm{I}$ site at the original (and now destroyed) SalI site. The $S p h I(1)-S p h I(2)$ fragment from pZC177 (deletion of which generates pZC1773) was now cloned back into pZC1776, and into pZC1773 as a control. In both cases, isolates were obtained with the SphI fragment cloned in both orientations; in pZC17731 and pZC17761, it was in the original orientation, and in pZC17732 and pZC17762 it was in the reverse orientation. The composition of the resulting plasmids are shown in Fig. 3b, together with their abilities to complement XC33, XS1D2 and XB25. Complementation of XC33 was as 
predicted in all cases. As regards complementation of the other E. coli auxotrophs, it is seen that pZC17731 behaved like pZC177, to which it should of course be identical. pZC17761 failed to complement XS1D2, though after $3 \mathrm{~d}$ incubation XS1D2(pZC17761) replicates on MinAmp began to show discrete $\mathrm{Arg}^{+}$colonies within the area of the replica. It complemented XB25, but growth was slower than for XB25(pZC1773), colonies taking $3 \mathrm{~d}$ to reach the size achieved by XB25(pZC1773) colonies in 1.5 d. pZC17732 and pZC17762 failed to complement $\mathrm{XS1D} 2$ or XB25. These results imply that the cloned $\arg C, J$ and $B$ genes are transcribed in $E$. coli onto a single transcript, so that the frameshift mutation created in argC has a polar effect on expression of $J$ and $B$. It seems likely therefore that the three genes have the same orientation. An alternative explanation which cannot as yet be completely ruled out is that ArgB and ArgJ polypeptides must interact with a functional ArgC polypeptide in order to function. However, such an obligatory interaction has not been found so far in any system, bacterial or otherwise.

\section{DISCUSSION}

We have shown above that plasmid pGX1 carries the $S$. coelicolor A3(2) argC, $J$ (assumed to be such from enzyme data), $B$ and $H$ genes within $17 \cdot 2 \mathrm{kbp}$ of insert DNA, and that $\mathrm{pZC177}$ carries the first three of these within about $5.8 \mathrm{kbp}$ in the order $C-J-B$. Even if the S. coelicolor A3(2) $\operatorname{argH}$ is not clustered with the other three, this points to a gene arrangement different from any other group. In $E$. coli, there is an $\operatorname{argECBH}$ cluster which is divergently transcribed from a common promoter-operator region between $E$ and $C$ (Glansdorff, 1987). In Bacillus, there is an early cluster $\operatorname{argCJBD}-c p a-F$ and an unlinked late cluster $\arg G H$ (Mountain et al., 1986; Sakanyan et al., 1992). As noted in the Introduction, $\arg G$ seems to be located on its own in $S$. coelicolor A3(2) and probably other streptomycetes; the positions of $\arg D$ and $F$ are uncertain, as are those of genes determining carbamoyl phosphate synthesis. The data of Ludovice et al. (1992) suggest that in $S$. clavuligerus also, $\arg C$ and $J$ are linked (assuming that their gene complementing E. coli argE is in fact an $\arg /)$.

A frameshift mutation produced in vitro had a polar effect on expression of $J$ and $B$, indicating that the latter two are transcribed in the same orientation as $\arg C$. It cannot of course be inferred that the three genes are co-transcribed in $S$. coelicolor A3(2); also, the suggestion that the three genes may be expressed in E. coli from their own promoter needs to be checked by comparative mapping of transcription starting points in the two organisms.

Our assay results of enzyme activities in extracts of XS1D2(pZC177) accord with those of Udaka (1966) in suggesting that streptomycetes possess an ornithine acetyltransferase, rather than a separate acetylglutamate synthase and acetylornithinase as in E. coli. That being so, it is not clear why the $S$. coelicolor A3(2) arg J gene fails to complement the $E$. coli $\arg A$ mutant XA4, whereas the complete cloned $B$. subtilis argJ does so (and the $3^{\prime}$ portion of the gene can complement $E$. coli $\arg E$ but not $\arg A$ : Mountain et al., 1986). The explanation may possibly lie in the nature of controls at the level of the various enzyme activities in the different species.

DNA sequencing indicates that the polypeptide product of S. coelicolor A3(2) argC is homologous to those of E. coli (Parsot et al., 1988), B. subtilis (Smith et al., 1991) and S. clavuligerus (Ludovice et al., 1992); the pairwise percentage identities of the predicted ArgC polypeptides are $S$. coelicolor A3(2)/E. coli, $42 \% ; S$. coelicolor A3(2)/B. subtilis, $36 \%$; and $S$. coelicolor $/ S$. clavuligerus, $67 \%$ in the 248 residues we have so far sequenced. The sequence just upstream of $S$. coelicolor A3(2) $\arg C$ shows a consensus promoter and putative ribosome binding site at appropriate positions, with a $6 \mathrm{bp}$ inverted repeat ACGTGC/GCACGT, hyphenated by $4 \mathrm{bp}$, lying between the proposed -35 and -10 promoter boxes. Perhaps more interestingly, an $18 \mathrm{bp}$ sequence ATTGCATAAACGTGCATC overlapping the -35 promoter box (see Fig. 4) shows $14 / 18$ identity (blocks 2-9 and 15-18 being completely identical) to a sequence within the 'ARG box' (ArgR repressor binding site) of the E. coli argR gene (Lim et al., 1987; Glansdorff, 1987), and shows 8/10 identity to the sequence ATTGAATTAA found within the B. subtilis argCO1 operator (Czaplewski et al., 1992). Similarly, Ludovice et al. (1992) have pointed out a sequence TTGATAAAGTGCAGTGATTTGTATAGTCA strongly resembling 'ARG boxes' in $E$. coli and B. subtilis. Recent work in our laboratory (A. Soutar, unpublished results) and that of P. Liras (M. Ludovice, P. Carrachas \& P. Liras, personal communication) accords with the idea that these sequence similarities are not fortuitous.

\section{ACKNOWLEDGEMENTS}

We thank members of the John Innes Institute for strains and plasmids, K. Devine and M. O'Reilly (Trinity College Dublin) for communicating unpublished data, and N. Glansdorff (Free University of Brussels) and P. Liras (University of Leon, Spain) and their colleagues for helpful discussions and the communication of results prior to publication, interaction between the Leeds and Leon groups being facilitated by grants under the EC Acciones Integradas programme. J. Baker and B. Merry helped with some of the experiments, and R. Nicholson and J. Swift with computer analysis of sequences. Z. H. acknowledges an SERC studentship.

\section{REFERENCES}

Adams, C. W., Fornwald, J. A., Schmidt, F. J., Rosenberg, M. \& Brawner, M. E. (1988). Gene organisation and structure of the Streptomyces lividans gal operon. J Bacteriol 170, 203-212.

Akrigg, D., Bleasby, A. J., Dix, N. I. M., Findlay, J. B. C., North, A. C. T., Parry-Smith, D., Wootton, J. C., Blundell, T. L., Gardner, S. P., Hayes, F., Islam, S., Sternberg, M. J. E., Thornton, J. M., Tickle, I. J. \& Murray-Rust, P. (1988). A protein sequence/structure database. Nature 335, 745.

Altenbucher, J. \& Eichenseer, C. (1991). A new system to study DNA amplifications in Streptomyces lividans. In Genetics and Product Formation in Streptomyces, pp. 253-263. Edited by S. Baumberg, H. Krügel \& D. Noack. New York \& London: Plenum Press.

Balbas, P., Soberon, X., Merino, E., Zurita, M., Lomeli, H., Valle Flores, N. \& Bolivar, F. (1986). Plasmid vector pBR322 and its special-purpose derivatives - a review. Gene 50, 3-40. 
Baumberg, S. (1981). The evolution of metabolic regulation. In Molecular and Cellular. 4spects of Microbial Evolution. 32nd Symposium of the Society for General Microbiology, pp. 229-272. Edited by M. J. Carlile, J. F. Collins \& B. E. B. Moseley. Cambridge: Cambridge University Press.

Birnboim, H. C. \& Doly, J. (1979). A rapid alkaline extraction procedure for screening recombinant plasmid DNA. Nucleic Acids Res 7, 1513-1523.

Bradford, M. M. (1976). A rapid and sensitive method for the quantitation of microgram quantities of protein utilizing the principle of protein-dye binding. Anal Biochem 72, 248-254.

Bretscher, A. P. \& Baumberg, S. (1976). Divergent transcription of the $\operatorname{argECBH}$ cluster of Escherichia coli K12. Mutations which alter control of enzyme synthesis. J Mol Biol 102, 205-220.

Cullum, J., Flett, F., Gravius, B., Hranueli, D., Miyashita, K., Pigac, J., Rauland, U. \& Redenbach, M. (1991). Analysis of amplifications and deletions in Streptomyces species. In Genetics and Product Formation in Streptomyces, pp. 265-272. Edited by S. Baumberg, H. Krügel \& D. Noack. New York \& London: Plenum Press.

Cunin, R., Glansdorff, N., Piérard, A. \& Stalon, V. (1986). Biosynthesis and metabolism of arginine in bacteria. Microbiol Rev 50, 314-352

Czaplewski, L. G., North, A. K., Smith, M. C. M., Baumberg, S. \& Stockley, P. G. (1992). Purification and initial characterization of AhrC: the regulator of arginine metabolism genes in Bacillus subtilis. Mol Microbiol 6, 267-275.

Davis, R. H. (1986). Compartmental and regulatory mechanisms in the arginine pathways of Neurospora crassa and Saccharomyces cerevisiae. Microbiol Rev 50, 280-313.

De Deken, R. E. (1963). Biosynthèse de l'arginine chez la levure. Biocbim Biophys Acta 78, 606-616.

Denes, G. (1970). Onithine acetyltransferase (Cblamydomonas reinbardtii). Methods Enøymol 17A, 271-275.

Glansdorff, N. (1987). Biosynthesis of arginine and polyamines. In Escherichia coli and Salmonella typhimurium: Cellular and Molecular Biology, pp. 321-344. Edited by F. C. Neidhardt. Washington, DC: American Society for Microbiology.

Hercomb, J., Thierbach, G., Baumberg, S. \& Parish, J. H. (1987). Cloning, characterization and expression in Eschericbia coli of a leucine biosynthetic gene from Streptomyces rocbei. J Gen Microbiol 133, 317-322.

Hindle, Z. (1990). A Study of Genes of Arginine Biosynthesis from Streptomyces. PhD. thesis, University of Leeds.

Hood, D. W., Heidstra, R., Swoboda, U. K. \& Hodgson, D. A. (1992). Molecular genetic analysis of proline and tryptophan biosynthesis in Streptomyces coelicolor A3(2): interaction between primary and secondary metabolism - a review. Gene 115, 5-12.

Hopwood, D. A., Bibb, M. J., Chater, K. F., Kieser, T., Bruton, C. J., Kieser, H. M., Lydiate, D. J., Smith, C. P., Ward, J. M. \& Schrempf, H. (1985). Genetic Manipulation of Streptomyces: a Laboratory Manual. Norwich: The John Innes Foundation.

Ishihara, H., Nakano, M. M. \& Ogawara, H. (1985). Cloning of a gene from Streptomyces species complementing arg $G$ mutations. $J$ Antibiot 38, 787-794.

Ishihara, H., Urabe, H., Kasama, H. \& Ogawara, H. (1991). Sequence of the gene encoding argininosuccinate synthetase in Streptomyces coelicolor A3(2). Actinomycetologia 5, 14-17.

Lim, C.-K., Smith, M. C. M., Petty, J., Baumberg, S. \& Wootton, J. C. (1989). Streptomyces griseus streptomycin phosphotransferase: expression of its gene in Eschericbia coli and sequence homology with other antibiotic phosphotransferases and with eukaryotic protein kinases. J Gen Microbiol 135, 3289-3302.
Lim, D., Oppenheim, J. D., Eckhardt, T. \& Maas, W. K. (1987). Nucleotide sequence of the argR gene of Escherichia coli K12 and isolation of its product, the arginine repressor. Proc Natl Acad Sci US A 84, 6697-6701.

Limauro, D., Avitabile, S., Cappellano, M., Puglia, A. M. \& Bruni, C. B. (1990). Cloning and characterisation of the histidine biosynthetic gene cluster of Streptomyces coelicolor A3(2). Gene 90, 31-41. Ludovice, M., Martin, J. F., Carrachas, P. \& Liras, P. (1992). Characterization of the Streptomyces clavuligerus argC gene encoding $\mathrm{N}$-acetylglutamyl-phosphate reductase: expression in Streptomyces lividans and effect on clavulanic acid production. J Bacteriol 174, 4606-4613.

Maniatis, T., Fritsch, E. F. \& Sambrook, J. (1982). Molecular Cloning : a Laboratory Manual. Cold Spring Harbor, NY : Cold Spring Harbor Laboratory.

Messing, J. \& Vieira, J. (1982). A new pair of M13 vectors for selecting either DNA strand of double digest testriction fragments. Gene 19, 269-276.

Mountain, A., Mann, N. H., Munton, R. N. \& Baumberg, S. (1984). Cloning of a Bacillus subtilis restriction fragment complementing auxotrophic mutants of eight Escherichia coli genes of arginine biosynthesis. Mol \& Gen Genet 197, 82-89.

Mountain, A., McChesney, J., Smith, M. C. M. \& Baumberg, S. (1986). Gene sequence encoding early enzymes of arginine synthesis within a cluster in Bacillus subtilis as revealed by cloning in Escherichia coli. J Bacteriol 165, 1026-1028.

Norrander, J., Kempe, T. \& Messing, J. (1983). Construction of improved M13 vectors using oligodeoxynucleotide-directed mutagenesis. Gene 26, 101-106.

Ogawara, H., Kasama, H., Nashimoto, K., Ohtsubo, M., Higashi, K. \& Urabe, H. (1993). Cloning, sequence and expression of the $\arg G$ gene from Streptomyces lavendulae. Gene 125, 91-96.

O'Reilly, M., Woodson, K., Dowds, B. C. A. \& Devine, K. M. (1994). The citrulline biosynthetic operon, $\arg C-F$, and a ribose transport operon, $r b s$, from Bacillus subtilis are negatively regulated by SpoOA. Mol Microbiol 11 (in the Press).

Padilla, G., Hindle, Z., Callis, R., Corner, A., Ludovice, M., Liras, P. \& Baumberg, S. (1991). The relationship between primary and secondary metabolism in streptomycetes. In Genetics and Product Formation in Streptomyces, pp. 35-45. Edited by S. Baumberg, H. Krügel \& D. Noack. New York and London: Plenum Press.

Parsot, C., Boyen, A., Cohen, G. N. \& Glansdorff, N. (1988). Nucleotide sequence of Escherichia coli $\arg B$ and $\arg C$ genes: comparison of $N$-acetylglutamate kinase and $N$-acetylglutamate- $\gamma$ semialdehyde dehydrogenase with homologous and analogous enzymes. Gene 68, 275-283.

Piendl, W., Kochl, S., Flett, F. \& Cullum, J. (1991). Analysis of large deletions and characterization of the deletion endpoints associated with an amplifiable DNA region in Streptomyces lividans. In Genetics and Product Formation in Streptomyces, pp. 273-281. Edited by S. Baumberg, H. Krügel \& D. Noack. New York \& London: Plenum Press.

Sakanyan, V., Kochikyan, A., Mett, I., Legrain, C., Charlier, D., Piérard, A. \& Glansdorff, N. (1992). A re-examination of the pathway for ornithine biosynthesis in a thermophilic and two mesophilic Bacillus species. J Gen Microbiol 138, 125-130.

Sakanyan, V., Charlier, D., Legrain, C., Kochikyan, A., Mett, I., Piérard, A. \& Glansdorff, N. (1993). Primary structure, partial purification and regulation of key enzymes of the acetyl cycle of arginine biosynthesis in Bacillus stearothermophilus: dual function of ornithine acetyltransferase. J Gen Microbiol 139, 393-402.

Schrempf, H. (1991). Genetic instability in Streptomyces. In Genetics 
and Product Formation in Streptomyces, pp. 245-252. Edited by S. Baumberg, H. Krügel \& D. Noack. New York \& London: Plenum Press.

Smith, C. P. \& Chater, K. F. (1988a). Cloning and transcription analysis of the entire glycerol utilization $(g y l A B X)$ operon of Streptomyces coelicalor A3(2) and the identification of a closely associated transcription unit. Mol \& Gen Genet 211, 129-137.

Smith, C. P. \& Chater, K. F. (1988b). Structure and regulation of controlling sequences for the Streptomyces coelicolor glycerol operon. $J$ Mol Biol 204, 569-580.

Smith, M. C. M., Mountain, A. \& Baumberg, S. (1990). Nucleotide sequence of the Bacillus subtilis argC gene encoding $N$ acetylglutamate- $\gamma$-semialdehyde dehydrogenase. Nucleic Acids Res $18,4595$.

Udaka, S. (1966). Pathway-specific pattern of control of arginine biosynthesis in bacteria. J Bacteriol 91, 617-621.

Vogel, H. J. \& Bonner, D. M. (1956). Acetylornithinase of Escherichia coli: partial purification and some properties. J Biol Chem 218, 97-106.

Received 16 August 1993; accepted 24 August 1993 